\title{
HEAT CONDUCTION IN AN INFINITE COMPOSITE SOLID WITH AN INTERFACE RESISTANCE
}

\author{
BY \\ W. A. MERSMAN
}

1. Introduction. The purpose of this paper is to solve the problem of onedimensional heat conduction in a body composed of two plane-boundary semi-infinite homogeneous solids of different materials in "imperfect contact" along their interface. ("Imperfect contact" is defined by equation (4) below.) The Laplace transformation method is used both in discovering and in rigorously establishing the solution, by means of the inversion theorems of Churchill and Doetsch( $\left.{ }^{1}\right)$. The question of uniqueness is not considered here.

2. The boundary value problem. Let $t$ denote time, $x$ the perpendicular distance from the interface, $a_{\nu}$ and $k_{\nu}$ the thermal diffusivities and conductivities, respectively, of the two materials, and $\lambda$ the interface resistance $\left.{ }^{2}\right)$. Throughout, $f(x)$ is a known function, integrable over any finite interval, such that

$$
|f(x)| \leqq \alpha \exp (\beta|x|)
$$

where $\alpha$ and $\beta$ are non-negative constants. If $U(x, t)$ is the temperature, we have the following boundary value $\operatorname{problem}\left({ }^{3}\right)$ :

$$
\begin{array}{rlrl}
\frac{\partial U}{\partial t} & =a_{v} \frac{\partial^{2} U}{\partial x^{2}}, & t>0, & x \neq 0, \\
\lim _{t \rightarrow 0} U(x, t) & =f(x), & & x \neq 0, \\
\lim _{x \rightarrow-0} k_{1} \frac{\partial U}{\partial x} & =\lim _{x \rightarrow+0} k_{2} \frac{\partial U}{\partial x}, & t>0, \\
U(+0, t)-U(-0, t) & =\lim _{x \rightarrow 0} \lambda k_{\nu} \frac{\partial U}{\partial x}, & t>0 .
\end{array}
$$

The constants, $a_{\nu}, k_{\nu}$, and $\lambda$ are all assumed to be greater than zero.

Presented to the Society, April 11, 1942; received by the editors November 22, 1941.

(1) Cf. Churchill, The solution of linear boundary-value problems in physics by means of the Laplace transformation. Part I. A theory for establishing a solution in the form of an integral, for problems with vanishing initial conditions, Math. Ann. vol. 114 (1937) pp. 591-613. This paper will be designated by [C].

Cf. Doetsch, Theorie und Anwendung der Laplace Transformation, Berlin, 1937. This book will be designated by [D].

(2) Cf. Riemann-Weber, Die Partiellen Differential-Gleichungen der Mathematischen Physik, 5 th edition, 1912 , vol. 2 pp. $85,100$.

(3) Throughout, $\nu=1$ if $x<0, \nu=2$ if $x>0$. 
Define $\Phi_{\nu}(t)$ as follows:

$$
\Phi_{1}(t)=U(-0, t), \quad \Phi_{2}(t)=U(+0, t),
$$

Then the solution can be written by means of $\Phi$ in the well known form( $\left(^{4}\right)$

$$
U(x, t)=V_{\nu}(x, t)+\int_{0}^{t} \Phi_{\nu}(t-\xi) \Psi\left(x a_{\nu}^{-1 / 2}, \xi\right) d \xi, \quad t>0
$$

where

$$
\begin{aligned}
\Psi(x, t) & =x \exp \left(-x^{2} / 4 t\right) / 2\left(\pi t^{3}\right)^{1 / 2}, \\
V_{1}(x, t) & =\frac{1}{2} \int_{-\infty}^{0} f(\xi)\left\{\Theta\left(x-\xi a_{1} t\right)-\Theta\left(x+\xi a_{1} t\right)\right\} d \xi, \\
\Theta(x, t) & =(\pi t)^{-1 / 2} \exp \left(-x^{2} / 4 t\right),
\end{aligned}
$$

and $V_{2}$ is obtained from $V_{1}$ upon replacing $a_{1}$ by $a_{2}$ and integrating from 0 to $+\infty$.

Equations (3)-(6) now give two simultaneous integral equations for the unknown functions $\Phi_{p}(t)$. These will be solved by the method of the Laplace transformation.

3. The transformed problem and its solution. Throughout we use the singly-infinite Laplace transformation:

$$
L\{U(x, t)\}=\int_{0}^{\infty} e^{-s t} U(x, t) d t=u(x, s) .
$$

For the sake of definiteness let $s$ be any complex number whose real part is greater than $32 \beta^{2}$. Denoting the transforms of $U, V$, and so on, by $u, v$, and so on, respectively, the transforms of equations (3), (4), (6), are:

$$
\begin{gathered}
\lim _{x \rightarrow-0} k_{1} \frac{\partial u(x, s)}{\partial x}=\lim _{x \rightarrow+0} k_{2} \frac{\partial u(x, s)}{\partial x}, \\
\phi_{2}(s)-\phi_{1}(s)=\lim _{x \rightarrow 0} \lambda k_{\nu} \frac{\partial u(x, s)}{\partial x}, \\
u(x, s)=\phi_{\nu}(s) \exp \left[-|x|\left(s / a_{\nu}\right)^{1 / 2}\right]+v_{\nu}(x, s) .
\end{gathered}
$$

Eliminating $\phi_{\nu}$ from these equations we obtain the transformed solution :

$$
u(x, s)=y_{\nu}(x, s)+w_{\nu}(x, s)
$$

(4) Cf. H. S. Carslaw, The mathematical theory of the conduction of heat in solids, 2d edition, London, 1921, \$\$18, 23.

(5) The first term of the right member is obtained by the "Faltung" rule. Cf. [D, chap. 8]. For the specific transformations used, see the table in [D, Appendix 2]. 
where

$$
\begin{aligned}
& y_{1}(x, s)=\left[k_{2} / a_{2}^{1 / 2}\left(A+B s^{1 / 2}\right)\right]\left\{\left(a_{2} s\right)^{-1 / 2} \int_{0}^{\infty} f(\xi)\right. \\
& \cdot \exp \left[-s^{1 / 2}\left|\xi a_{2}^{-1 / 2}-x a_{1}^{-1 / 2}\right|\right] d \xi \\
& \left.-\left(a_{1} s\right)^{-1 / 2} \int_{-\infty}^{0} f(\xi) \dot{\exp }\left[-|x+\xi|\left(s / a_{1}\right)^{1 / 2}\right] d \xi\right\}, \quad x<0, \\
& A=k_{1} a_{1}^{-1 / 2}+k_{2} a_{2}^{-1 / 2}, \quad B=\lambda k_{1} k_{2}\left(a_{1} a_{2}\right)^{-1 / 2}, \\
& w_{1}(x, s)=\left[1 / 2\left(a_{1} s\right)^{1 / 2}\right] \int_{-\infty}^{0} f(\xi)\left\{\exp \left[-|x-\xi|\left(s / a_{1}\right)^{1 / 2}\right]\right. \\
& \left.+\exp \left[-|x+\xi|\left(s / a_{1}\right)^{1 / 2}\right]\right\} d \xi, \\
& x<0 \text {, }
\end{aligned}
$$

and $y_{2}, w_{2}$ are obtained from $y_{1}, w_{1}$, respectively, upon interchanging the subscripts 1 and 2 and the integrals $\int_{0}^{\infty}$ and $\int_{-\infty}^{0}$.

4. The inversion problem. It is easily seen that the operations $\partial / \partial x$ and $\lim _{x \rightarrow 0}$ are interchangeable with the integrations in (11) and (13), and hence that (10) is an actual solution of the boundary value problem $\left(3^{\prime}\right)$ and $\left(4^{\prime}\right)$. To show that the inverse Laplace transform of $u(x, s)$ exists and is a solution of the original boundary value problem, we use the inversion theorems of Doetsch and Churchill. For the inverse we use the notation:

$$
L^{-1}\{u(x, s)\}=\lim _{\omega \rightarrow \infty} \frac{1}{2 \pi i} \int_{\gamma-\omega i}^{\gamma+\omega i} e^{t s} u(x, s) d s
$$

that is, $L^{-1}$ always means this particular form of the inverse, whether or not it is true that $u(x, s)=L\left\{L^{-1}[u(x, s)]\right\}$. Throughout, $\gamma>32 \beta^{2}, R\left(s^{1 / 2}\right) \geqq 0$.

Note that $y$ and $w$ are composed of two types of integrals, according as $x$ and $\xi$ have the same or opposite signs. It is sufficiently general to consider the following:

$$
\begin{array}{ll}
z(x, s)=s^{-1} \int_{0}^{\infty} f(\xi) \exp \left[-(x+\xi) s^{1 / 2}\right] d \xi, & x \geqq 0, \\
g(x, s)=s^{-1 / 2} \int_{0}^{\infty} f(\xi) \exp \left[-|x-\xi| s^{1 / 2}\right] d \xi, & x \geqq 0 .
\end{array}
$$

We begin with some inequalities. Noting that $\left|e^{\zeta}\right|=\exp [R(\zeta)]$ and that $R\left(s^{1 / 2}\right)=[(R(s)+|s|) / 2]^{1 / 2}$, the following are easily obtained:

$$
\begin{array}{rlr}
\left|s^{n} z(x, s)\right| \leqq 2^{3 / 2} \alpha|s|^{n-3 / 2} \exp \left[-x(|s| / 2)^{1 / 2}\right], & & n \geqq 0 . \\
\left|\frac{\partial^{n} g(x, s)}{\partial x^{n}}\right| \leqq 3 \alpha(2)^{1 / 2} e^{\beta x}|s|^{-1+n / 2}, & n=0,1 .
\end{array}
$$




$$
\begin{aligned}
& \int_{\xi_{1}}^{\infty}\left|s^{(n-1) / 2} \exp \left[t s-|\xi-x| s^{1 / 2}\right] f(\xi)\right| d \xi \\
& \leqq 2^{3 / 2} \alpha|s|^{-1+n / 2} \exp \left[t \gamma-\xi_{1}(|s| / 2)^{1 / 2} / 4\right], \\
& \gamma=R(s), \quad \xi_{1}>4 x \geqq 0, n=0,1 .
\end{aligned}
$$

By means of these inequalities the following lemmas can be proved:

LEMMA 1. If $0<x_{1} \leqq x \leqq x_{2}, 0 \leqq t \leqq t_{1}, n=0,1$, then $L^{-1}\left\{s^{n} z(x, s)\right\}$ and $L^{-1}\left\{\partial^{2} z(x, s) / \partial x^{2}\right\}$ exist and converge uniformly in $x$ and $t$.

This follows immediately from inequality (16).

LEMMA 2. If $0 \leqq x \leqq x_{1}, 0<t_{1} \leqq t \leqq t_{2}$, then

$$
L^{-1}\{g(x, s)\}
$$

exists and converges uniformly in $x$ and $t$.

Proof. We wish to show that, given any $\epsilon>0$, there exists an $\Omega(\epsilon)$, independent of $x$ and $t$ such that, if $\omega_{2}>\omega_{1}>\Omega$, then

$$
\left|\int_{\gamma-\omega_{2} i}^{\gamma-\omega_{1} i} e^{t s} g(x, s) d s+\int_{\gamma+\omega_{1} i}^{\gamma+\omega_{2} i} e^{t s} g(x, s) d s\right|<\epsilon .
$$

By equation (15) and inequality (18), the order of integration can be inverted. Hence, consider

$$
\begin{aligned}
\mid \int_{0}^{\infty} f(\xi) \int_{\gamma-\omega_{2} i}^{\gamma-\omega_{1} i} s^{-1 / 2} \exp \left[t s-|\xi-x| s^{1 / 2}\right] d s d \xi \\
\quad+\int_{0}^{\infty} f(\xi) \int_{\gamma+\omega_{1} i}^{\gamma+\omega_{2} i} s^{-1 / 2} \exp \left[t s-|\xi-x| s^{1 / 2}\right] d s d \xi \mid .
\end{aligned}
$$

Since the integrands are analytic in $s$, the integrations on $s$ can be carried out over the contour $C_{1}+C_{2}+C_{3}+C_{4}+C_{5}+C_{6}$, where the $C^{\prime}$ 's are straight line segments with end points as follows: $C_{1}: \gamma-\omega_{2} i,-\omega_{2} i ; C_{2}:-\omega_{2} i,-\omega_{1} i$; $C_{3}:-\omega_{1} i, \gamma-\omega_{1} i ; C_{4}: \gamma+\omega_{1} i, \omega_{1} i ; C_{5}: \omega_{1} i, \omega_{2} i ; C_{6}: \omega_{2} i, \gamma+\omega_{2} i$.

The contribution of $C_{1}$ is

$$
\int_{0}^{\infty} f(\xi) \int_{\gamma}^{0}\left(\zeta-\omega_{2} i\right)^{-1 / 2} \exp \left[t \zeta-\omega_{2} i-|\xi-x|\left(\zeta-\omega_{2} i\right)^{1 / 2}\right] d \zeta d \xi,
$$

which is less in absolute value than

$$
\int_{0}^{\infty} \alpha \Omega^{-1 / 2} \exp \left[\beta \xi+t \gamma-|\xi-x|(\Omega / 2)^{1 / 2}\right] \gamma d \xi \leqq \frac{3 \alpha(2)^{1 / 2} e^{t \gamma+\beta x} \gamma}{\Omega} .
$$

A similar proof shows that the total contribution of $C_{1}+C_{3}+C_{4}+C_{6}$ is less than 


$$
\frac{12 \alpha \gamma(2)^{1 / 2} e^{t \gamma+\beta x}}{\Omega},
$$

which approaches zero as $\Omega$ approaches infinity, uniformly in $x$ and $t$.

Finally, the contributions of $C_{2}$ and $C_{5}$ can be combined to give

$$
\begin{aligned}
2 \int_{0}^{\infty} f(\xi) \int_{\omega_{1}}^{\omega_{2}} \exp \left[-|\xi-x|(\eta / 2)^{1 / 2}\right] & \\
\therefore & \cdot \cos \left[t \eta-(\pi / 4)-|\xi-x|(\eta / 2)^{1 / 2}\right] \eta^{-1 / 2} d \eta d \xi .
\end{aligned}
$$

Split the range of integration at $\xi=\xi_{1}>2 x_{1}$. The contribution of the integral from $\xi_{1}$ to $\infty$ is less in absolute value than

$$
2 \int_{\xi_{1}}^{\infty} \alpha e^{\beta \xi} \int_{\omega_{1}}^{\omega_{2}} \eta^{-1 / 2} \exp \left[-\xi(\eta / 2)^{1 / 2} / 2\right] d \eta d \xi .
$$

On making the change of variable $\eta=8 \zeta^{2} \xi^{-2}$ this is found to be less than

$$
8 \alpha(2)^{1 / 2} \int_{\xi_{1}}^{\infty} \xi^{-1} \exp \left[\beta \xi-\xi(\Omega / 2)^{1 / 2} / 2\right] d \xi .
$$

If $\Omega>8 \beta^{2}$ this integral converges, and hence can be made less than $\epsilon$ by a suitable choice of $\xi_{1}$. Choose such a $\xi_{1}$ and let it remain fixed.

We have remaining the integral from 0 to $\xi_{1}$ :

$$
\begin{aligned}
\int_{0}^{\xi_{1}} f(\xi) \int_{\omega_{1}}^{\omega_{2}} \eta^{-1 / 2} \exp \left[-|\xi-x|(\eta / 2)^{1 / 2}\right] \\
\quad \cos \left[t_{\eta}-(\pi / 4)-|\xi-x|(\eta / 2)^{1 / 2}\right] d \eta d \xi .
\end{aligned}
$$

On making the substitution

$$
(t \eta)^{1 / 2}-|\xi-x| / 2(2 t)^{1 / 2}=\zeta
$$

and using the addition formula for the cosine, this is seen to be less in absolute value than

$$
\begin{aligned}
2 t^{-1 / 2} \mid \int_{0}^{\xi_{1}} f(\xi) \exp \left[-(\xi-x)^{2} / 4 t\right] \cos \left[(\pi / 4)+(\xi-x)^{2} / 8 t\right] \\
\\
\cdot \int_{\zeta_{1}}^{\zeta_{2}} \exp \left[-\zeta|\xi-x| /(2 t)^{1 / 2}\right] \cos \zeta^{2} d \zeta d \xi
\end{aligned}
$$

plus the same expression with cos replaced by sin, where

$$
\zeta_{n}=\left(\omega_{n}\right)^{1 / 2}-|\xi-x| / 2(2 t)^{1 / 2}, \quad n=1,2 .
$$

By the second mean-value theorem this is equal to 


$$
\begin{aligned}
2 t^{-1 / 2} \mid \int_{0}^{\xi_{1}} f(\xi) \exp \left[-|\xi-x|\left(\omega_{1} / 2\right)^{1 / 2}\right] \cos \left[(\pi / 4)+(\xi-x)^{2} / 8 t\right] & \cdots \\
& \cdot \int_{\zeta_{1}}^{\zeta_{3}} \cos \zeta^{2} d \zeta d \xi \mid
\end{aligned}
$$

plus the same expression with cos replaced by sin, and $\zeta_{1} \leqq \zeta_{3} \leqq \zeta_{2}$.

But the integrals $\int_{0}^{\infty} \sin \zeta^{2} d \zeta$ and $\int_{0}^{\infty} \cos \zeta^{2} d \zeta$ converge; hence there is a positive number $M$ such that

$$
\left|\int_{\zeta_{i}}^{\zeta_{3}} \cos \zeta^{2} d \zeta\right|<M>\left|\int_{\zeta_{1}}^{\zeta_{3}} \sin \zeta^{2} d \zeta\right|
$$

for any values of $\zeta_{1}, \zeta_{3}$. Thus the expression (19) is less than

$$
4 M t^{-1 / 2} \int_{0}^{\xi_{1}}|f(\xi)| \exp \left[-|\xi-x|\left(\omega_{1} / 2\right)^{1 / 2}\right] d \xi .
$$

On splitting the range of integration at $\xi=x$ this is seen to be less than

$$
12(2)^{1 / 2} M e^{b x} /(t \Omega)^{1 / 2}
$$

which approaches zero as $\Omega$ approaches infunity, uniformly in $x$ and $t$, q.e.d.

LEMMA 3. If $0 \leqq x \leqq x_{1}, 0<t_{1} \leqq t \leqq t_{2}$, then $L^{-1}\{\partial g(x, s) / \partial \dot{x}\}$ exists and converges uniformly in $x$ and $t$.

Proof. Using the method and notation of the proof of Lemma 2, it is easily found that the contribution of $C_{1}+C_{3}+C_{4}+C_{6}$ is less in absolute value than

$$
12 \alpha \gamma e^{t \gamma+\beta x}(2 / \Omega)^{1 / 2}
$$

Similarly, the contribution of $C_{2}+C_{5}$ over the range $\xi_{1} \leqq \xi \leqq \infty$ is less than

$$
96 \alpha \xi_{1}^{-1} \exp \left[-\xi_{1}(\Omega / 2)^{1 / 2} / 4\right] \text {. }
$$

Choose a fixed $\xi_{1}$ such that this is less than $\epsilon$. Then we have remaining the expression

$$
\begin{aligned}
2 t^{-1} \int_{0}^{\xi_{1}}|f(\xi)| & \mid \int_{\zeta_{1}}^{\zeta_{2}} \exp \left[-|\xi-x|\left\{\zeta+|\xi-x| / 2(2 t)^{1 / 2}\right\} /(2 t)^{1 / 2}\right] \\
& \cdot \cos \left[\zeta^{2}-(\xi-x)^{2} / 8 t\right] \cdot\left[\zeta+|\xi-x| / 2(2 t)^{1 / 2}\right] d \zeta \mid d \xi
\end{aligned}
$$

By means of the last bracket this can be separated into two terms, the second of which, as in the proof of Lemma 2, is less than

$$
12 \alpha M \xi_{1} e^{\beta x}\left(2 / t^{3} \Omega\right)^{1 / 2} .
$$

This leaves: 
$2 \alpha t^{-1} \int_{0}^{\xi_{1}} e^{\beta \xi} \mid \int_{\zeta_{1}}^{\zeta_{2}} \exp \left[-|\xi-x|\left\{\zeta+|\xi-x| / 2(2 t)^{1 / 2}\right\} /(2 t)^{1 / 2}\right]$

$$
\cdot \cos \left[\zeta^{2}-(\xi-x)^{2} / 8 t\right] \zeta d \zeta \mid d \xi
$$

Integrating by parts with respect to $\zeta$ gives

$$
\begin{array}{r}
\alpha t^{-1} \int_{0}^{\xi_{1}} e^{\beta \xi}\left\{\exp \left[-|\xi-x|\left(\omega_{2} / 2\right)^{1 / 2}\right]+\exp \left[-|\xi-x|\left(\omega_{1} / 2\right)^{1 / 2}\right]\right\} d \xi \\
+\alpha\left(2 t^{3}\right)^{-1 / 2} \int_{0}^{\xi_{1}} e^{\beta \xi}\left|\int_{\xi_{1}}^{\xi_{2}}\right| \xi-x \mid \exp \left[-|\xi-x|\left\{\zeta+|\xi-x| / 2(2 t)^{1 / 2}\right\} /(2 t)^{1 / 2}\right] \\
\cdot \sin \left[\zeta^{2}-(\xi-x)^{2} / 8 t\right] d \xi \mid d \xi .
\end{array}
$$

which, as before, is less than

$$
6 \alpha e^{\beta x} t^{-1}(2 / \Omega)^{1 / 2}+6 \alpha M \xi_{1} e^{\beta x} /\left(t^{3} \Omega\right)^{1 / 2},
$$

which approaches zero as $\Omega$ approaches infinity, uniformly in $x$ and $t$, q.e.d.

The following can be proved by the same methods:

LEMMA 4. If $0 \leqq x \leqq x_{1}, 0<t_{1} \leqq t \leqq t_{2}, n=0,1$, then $L^{-1}\left\{\partial^{n} z(x, s) / \partial x^{n}\right\}$ exists and converges uniformly in $x$ and $t$.

Since $|s g(x, s)|$ does not approach zero as $|s|$ approaches infinity, the Laplace transformation method cannot be used to establish the existence ${ }^{\circ}$ ) of $\partial G / \partial t$ and $\partial^{2} G / \partial x^{2}$. This will be done by classical methods by means of the following:

LEMMA 5. $g(x, s)=L\{G(x, t)\}$, where

$$
G(x, t)=\int_{0}^{\infty} f(\xi) \Theta(x-\xi, t) d \xi,
$$

and then $G(x, t)=L^{-1}\{g(x, s)\}$.

Proof. The substitution $\xi=x+2 \zeta t^{1 / 2}$ shows that

$$
\int_{0}^{\infty}|f(\xi) \Theta(x-\xi, t)| d \xi \leqq 2 \alpha \exp \left[\beta x+\beta^{2} t\right]
$$

Hence $L\{G\}$ exists and converges absolutely. By (20) the order of integration in $L\{G\}$ can be inverted, giving $\left({ }^{7}\right)$

() Cf. [C, Theorem 6].

(?) Cf. [D, Appendix 2, table]. 


$$
L\{G\}=\int_{0}^{\infty} f(\xi) L\{\Theta(x-\xi, t)\} d \xi=g(x, s) .
$$

Hence, by [D, chap. $6, \S 5$, Theorem 2 ], $G$ is given by the inversion integral $L^{-1}\{g\}$, q.e.d.

Using these lemmas we are now in a position to establish the solution of the original boundary value problem.

\section{The solution established.}

THEOREM 1. The function $W_{\nu}(x, t) \equiv L^{-1}\left\{w_{\nu}(x, s)\right\}$ exists and has the following properties:

(a) $W_{1}(x, t)=(1 / 2) \int_{-\infty}^{0} f(\xi)\left\{\Theta\left(x-\xi, a_{1} t\right)+\Theta\left(x+\xi, a_{1} t\right)\right\} d \xi$ and $W_{2}$ is obtained from $W_{1}$ upon replacing $a_{1}$ by $a_{2}$ and integrating from 0 to $+\infty$.

(b) $W_{\nu}(x, t)$ satisfies the differential equation (1).

(c) $W_{\nu}(x, t)$ satisfies the initial condition (2) at any point of continuity of $f(x)$.

(d) $\lim _{x \rightarrow 0} \partial W_{v} / \partial x=0$.

Proof. Equation (a) follows from Lemma 5, and the remainder of the theorem is then easily proved by classical methods $\left({ }^{8}\right)$.

To obtain the complete solution of the problem it is now sufficient to prove the following:

THEOREM 2. The function $Y_{\nu}(x, t) \equiv L^{-1}\left\{y_{\nu}(x, s)\right\}$ exists and has the following properties:

(a) $Y_{\nu}(x, t)$ satisfies the differential equation (1).

(b) $\lim _{t \rightarrow 0} Y_{r}(x, t)=0$, $x \neq 0$.

(c) $\lim _{x \rightarrow-0} k_{1} \frac{\partial Y_{1}}{\partial x}=\lim _{x \rightarrow+0} k_{2} \frac{\partial Y_{2}}{\partial x}$, $t>0$.

(d) $\quad Y_{2}(+0, t)+W_{2}(+0, t)-Y_{1}(-0, t)-W_{1}(-0, t)=\lim _{x \rightarrow 0} \lambda k_{\nu} \frac{\partial Y_{\nu}}{\partial x}, \quad t>0$.

Proof. $Y_{\nu}$ exists by Lemma 1.

(a) By Lemma 1 and inequality (16), $Y$, satisfies the hypotheses of [C, Theorems 6 and 9]. Hence, if $x \neq 0, t>0, \partial Y_{v} / \partial t=L^{-1}\left\{s y_{\nu}\right\}$. But $\partial^{2} Y_{v} / \partial x^{2}$ $=L^{-1}\left\{\partial^{2} y_{v} / \partial x^{2}\right\}$, and, since (11) can be differentiated inside the integral sign,

$$
\frac{\partial^{2} y_{v}}{\partial x^{2}}=a_{v}^{-1} s y_{v}, \text { q.e.d. }
$$

(b) By Lemma 1 and inequality (16) the hypotheses of [C, Theorem 4] are satisfied, from which the result follows immediately.

( 8 C. Carslaw, loc. cit., $\$ \$ 16-18$ for a proof under more stringent conditions on $f(x)$. 
(c) and (d) From equations (11) and (13) it is easily seen that

$$
\begin{gathered}
\lim _{x \rightarrow-0} k_{1} \frac{\partial y_{1}(x, s)}{\partial x}=\lim _{x \rightarrow+0} k_{2} \frac{\partial y_{2}(x, s)}{\partial x}, \\
y_{2}(+0, s)+w_{2}(+0, s)-y_{1}(-0, s)-w_{1}(-0, s)=\lim _{x \rightarrow 0} \lambda k_{v} \frac{\partial y_{v}}{\partial x}
\end{gathered}
$$

By Lemma (2) and inequality (17) $w_{\nu}(x, s)$ satisfies the hypotheses of [C, Theorem 8]. Hence $W \cdot( \pm 0, t)=L^{-1}\left\{w_{\nu}( \pm 0, s)\right\}, t>0$.

By Lemma 4 and inequality (16), $y_{\nu}(x, s)$ and $\partial y_{\nu} / \partial x$ satisf.y the hypotheses $\left({ }^{9}\right)$ of $\left[\mathrm{C}\right.$, Theorems 8 and 9 ]. Hence $Y_{\nu}( \pm 0, t)=L^{-1}\left\{y_{v}( \pm 0, s)\right\}, t>0$ and

$$
\lim _{x \rightarrow \pm 0} \frac{\partial Y_{\nu}}{\partial x}=L^{-1}\left\{\lim _{x \rightarrow \pm 0} \frac{\partial y_{\nu}}{\partial x}\right\}
$$

Hence conditions (c) and (d) follow from (21) and (22), q.e.d.

To summarize, the solution of the boundary value problem (1)-(4) is given by

$$
U(x, t)=W_{\nu}(x, t)+Y_{\nu}(x, t)
$$

where

$$
W_{1}(x, t)=\frac{1}{2} \int_{-\infty}^{0} f(\xi)\left\{\Theta\left(x-\xi, a_{1} t\right)+\Theta\left(x+\xi, a_{1} t\right)\right\} d \xi
$$

$W_{2}(x, t)$ is obtained from $W_{1}(x, t)$ upon replacing $a_{1}$ by $a_{2}$ and integrating from $\theta$ to $+\infty, \Theta$ is given by (9), and

$$
Y_{\nu}(x, t)=\lim _{\omega \rightarrow \infty} \frac{1}{2 \pi i} \int_{\gamma-\omega i}^{\gamma+\omega i} e^{t s} y_{\nu}(x, s) d s,
$$

if $\gamma>32 \beta^{2}$, where $y_{\nu}(x, s)$ is given by equations (11).

6. Explicit forms of $Y$. In the preceding section the function $Y_{\nu}(x, t)$ was obtained as a complex inversion integral. We now develop two more practical formulas.

THEOREM 3. $Y_{v}(x, t)$ is given by the following formulas:

$$
\begin{array}{r}
Y_{1}(x, t)=k_{2} a_{2}^{-1 / 2} \int_{0}^{\infty} e^{-A \mu}\left\{a_{2}^{-1 / 2} \int_{0}^{\infty} f(\xi) \Theta\left(\left|\xi a_{2}^{-1 / 2}-x a_{1}^{-1 / 2}\right|+B \mu, t\right) d \xi\right. \\
\left.-a_{1}^{-1 / 2} \int_{-\infty}^{0} f(\xi) \Theta\left(B \mu+|x+\xi| a_{1}^{-1 / 2}, t\right) d \xi\right\} d \mu, \quad x<0<t,
\end{array}
$$

(9) Actually, in [C] it is assumed that $\partial y(x, s) / \partial x=O\left(|s|^{-1}\right)$, but the weaker condition $\partial y / \partial x=O\left(|s|^{-1 / 2}\right)$ given by our inequality (16), is sufficient, as can be seen from an examination of Churchill's proof. 
and $Y_{2}$ is obtained from $Y_{1}$ upon interchanging the subscripts 1 and 2 and the integrals $\int_{0}^{\infty} d \xi$ and $\int_{-\infty}^{0} d \xi$.

Proof. Note that in equations (11) each term is of the form

$$
j(x, s) \equiv s^{-1 / 2}\left(A+B s^{1 / 2}\right)^{-1} \int_{0}^{\infty} f(\xi) \exp \left[-(x+\xi) s^{1 / 2}\right] d \xi, \quad x>0 .
$$

Since $\left(A+B s^{1 / 2}\right)^{-1}=\int_{0}^{\infty} \exp \left[-\left(A+B s^{1 / 2}\right) \mu\right] d \mu$, we can write

$$
j(x, s)=\int_{0}^{\infty} e^{-A \mu} \int_{0}^{\infty} f(\xi) s^{-1 / 2} \exp \left[-(x+\xi+B \mu) s^{1 / 2}\right] d \xi d \mu .
$$

By Lemma 1, $J(x, t)=L^{-1}\{j(x, s)\}$ exists. Hence, to prove the present theorem it is sufficient to show that the integration on $s$ can be performed inside the inner integral in (25). This will be done in two steps.

First we show that the integrations on $\mu$ and $s$ can be inverted. It is sufficient to prove that $\left({ }^{10}\right)$

$$
\lim _{\mu_{1} \rightarrow \infty} \int_{0}^{\mu_{1}} e^{-A \mu} e^{t_{s}} \int_{0}^{\infty} s^{-1 / 2} f(\xi) \exp \left[-(x+\xi+B \mu) s^{1 / 2}\right] d \xi d \mu
$$

converges uniformly in $s$, and

$$
\lim _{\omega \rightarrow \infty} \int_{\gamma-\omega i}^{\gamma+\omega i} e^{-A \mu} e^{t s} \int_{0}^{\infty} s^{-1 / 2} f(\xi) \exp \left[-(x+\xi+B \mu) s^{1 / 2}\right] d \xi d s
$$

converges uniformly in $\mu$.

To prove (26) note that the absolute value of the integral from $\mu_{1}$ to $\infty$ is less than

$$
\int_{\mu_{1}}^{\infty} e^{-A \mu} e^{\ell \gamma} 2^{3 / 2} \alpha|s|^{-1} \exp \left[-(x+B \mu)(|s| / 2)^{1 / 2}\right] d \mu
$$

by inequality (16), with $n=1 / 2$ and $x$ replaced by $x+B \mu$. But (28) is less than

$$
\frac{2^{3 / 2} \alpha e^{t \gamma}|s|^{-1} \exp \left[-x(|s| / 2)^{1 / 2}\right] \exp \left[-\left\{A+B(|s| / 2)^{1 / 2}\right\} \mu_{1}\right]}{A+B(|s| / 2)^{1 / 2}},
$$

which obviously approaches zero as $\mu_{1}$ approaches infinity, uniformly in $s$.

To prove (27), the absolute value of the double integral is less than

$$
\int_{\gamma-\infty i}^{\gamma+\infty i} 2^{3 / 2} \alpha e^{t \gamma}|s|^{-1} \exp \left[-x(|s| / 2)^{1 / 2}\right] d s
$$

by inequality (16). But this converges and is independent of $\mu$, q.e.d. Hence

(10) Cf. Pierpont, Theory of functions of real variables, 1905, vol. 1 p. $489 \$ 680.3$. 
$J(x, t)=\int_{0}^{\infty} e^{-A \mu} \lim _{\omega \rightarrow \infty} \frac{1}{2 \pi i} \int_{\gamma-\omega i}^{\gamma+\omega i} e^{t s} s^{-1 / 2} \int_{0}^{\infty} f(\xi) \exp \left[-(x+\xi+B \mu) s^{1 / 2}\right] d \xi d s d \mu$.

Now, by a proof similar to that of Lemma 5, or by inequalities (16) and (18), the inversion of the integrations on $\xi$ and $s$ can be justified. Therefore

$$
J(x, t)=\int_{0}^{\infty} e^{-\Lambda \mu} \int_{0}^{\infty} f(\xi) L^{-1}\left\{s^{-1 / 2} \exp \left[-(x+\xi+B \mu) s^{1 / 2}\right]\right\} d \xi d \mu .
$$

But the inner integral is well known(11). Hence,

$$
J(x, t)=\int_{0}^{\infty} e^{-\Lambda \mu} \int_{0}^{\infty} f(\xi) \Theta(x+\xi+B \mu, t) d \xi d \mu, \text { q.e.d. }
$$

Another form of $Y$ is given by the following

TheOREM 4.

$$
\begin{aligned}
Y_{1}(x, t)= & \left(k_{2} / B a_{2}^{1 / 2}\right) \int_{0}^{t} \exp \left[A^{2}(t-\tau) B^{-2}\right] \\
& \cdot\left\{a_{2}^{-1 / 2} \int_{0}^{\infty} f(\xi) \Psi\left(\xi a_{2}^{-1 / 2}-x a_{1}^{-1 / 2}, \tau\right) d \xi\right. \\
& +a_{1}^{-1 / 2} \int_{-\infty}^{0} f(\xi) \Psi\left([x+\xi] a_{1}^{-1 / 2}, \tau\right) d \xi \\
& -\left(A / B a_{2}^{1 / 2}\right) \int_{0}^{\infty} f(\xi) \Theta\left(\xi a_{2}^{-1 / 2}-x a_{1}^{-1 / 2}, \tau\right) d \xi \\
& \left.+A B^{-1} \int_{-\infty}^{0} f(\xi) \Theta\left(x+\xi, a_{1} \tau\right) d \xi\right\} d \tau, \quad x<0<t,
\end{aligned}
$$

and $Y_{2}(x, t)$ is obtained from $Y_{1}(x, t)$ on interchanging subscripts 1 and 2 and the integrals $\int_{0}^{\infty}$ and $\int_{-\infty}^{0}$.

Proof. On observing that $\left(A+B s^{1 / 2}\right)^{-1}=\left(s-A^{2} B^{-2}\right)^{-1}\left[s^{1 / 2} B^{-1}-A B^{-2}\right]$, and that $L^{-1}\left\{\left(s-A^{2} B^{-2}\right)^{-1}\right\}=\exp \left[A^{2} t B^{-2}\right]$, the result follows immediately from the "Faltung theorem( $\left.{ }^{12}\right)$ " since all the Laplace transformations concerned are absolutely convergent. (Compare the proof of Lemma 5 above.)

University of California,

$$
\text { Davis, Calif. }
$$

(11) Cf. [D, Appendix 2, table].

(12) Cf. [D, chap. 8. 85, Theorem IV $\mathrm{b}$. 